\title{
Characteristics of Nur77 and its ligands as potential anticancer compounds (Review)
}

\author{
LINGJUAN WU and LIQUN CHEN
}

College of Biological Science and Engineering, Fuzhou University, Fuzhou, Fujian 350108, P.R. China

Received March 12, 2018; Accepted September 5, 2018

DOI: $10.3892 / \mathrm{mmr} .2018 .9515$

\begin{abstract}
Nuclear receptor subfamily 4 group A member 1 (NR4A1; also termed Nur77/TR3/NGFIB), a member of the nuclear receptor superfamily, is expressed as an early response gene to regulate the expression of multiple target genes. Nur77 has the typical structure of a nuclear receptor, including an N-terminal domain, a DNA binding domain, and a ligand-binding domain. The expression and localization of Nur77 are closely associated with its roles in cell proliferation and apoptosis. Nur77 was first identified as an orphan receptor, the endogenous ligand of which has not yet been identified; however, an increasing number of compounds targeting Nur77 have been reported to have beneficial effects in the treatment of cancer and other diseases. This review provides a brief overview of the identification, structure, expression and localization, transcriptional role and non-genomic function of Nur77, and summarizes the ligands that have been shown to interact with Nur77, including cytosporone B, cisplatin, TMPA, PDNPA, CCE9, THPN, Z-ligustilide, celastrol and bisindole methane compounds, which may potentially be used to treat cancer in humans.
\end{abstract}

\section{Contents}

1. Introduction

2. Identification of Nur77

3. Structure, expression and localization of Nur77

4. Genomic and non-genomic functions of the orphan receptor Nur77

5. Compounds that modulate the regulation of Nur77

6. Conclusions and perspectives

Correspondence to: Dr Liqun Chen, College of Biological Science and Engineering, Fuzhou University, 2 Xueyuan Road, Fuzhou, Fujian 350108, P.R. China

E-mail: lqchen@fzu.edu.cn

Key words: nuclear receptor, Nur77, cancer, compounds, target therapy

\section{Introduction}

Nuclear receptors (NRs) are members of a large superfamily of transcription factors in metazoans, which are involved in complex biological processes and are major pharmacological targets (1-3). The dysfunction of NR activities is associated with a range of diseases, including diabetes, Alzheimer's disease, cancer and cardiovascular disease (4). NRs are intracellular signaling proteins that bind to specific ligands, particularly small lipid-soluble molecules, including steroids, thyroxine, retinoic acid and vitamin D.

Structurally, NRs are typically divided into five regions: $\mathrm{A} / \mathrm{B}$ region, $\mathrm{C}$ region, $\mathrm{D}$ region, $\mathrm{E}$ region and $\mathrm{F}$ region (Fig. 1A). The main domains of NRs are the N-terminal domain, the conserved DNA-binding domain (DBD) and the C-terminal ligand-binding domain (LBD) (5). The A/B region, which contains the ligand-independent activation function 1 (AF-1) domain, has been described as intrinsically disordered, and is involved in protein-protein interactions and receptor functions (6). In the $C$ region, the general protein structure is similar among NRs $(7,8)$. The DBD within the $\mathrm{C}$ region includes the zinc finger transcription factor motif, in which eight conserved cysteine residues coordinate with two zinc ions and mediate homodimerization or heterodimerization (2). Region D is variable, with a flexible hinge region that connects the $\mathrm{C}$ and $\mathrm{E}$ regions. This region often contains a nuclear localization sequence (NLS) and can alter the structure of the protein, including increasing the flexibility of the protein $(5,9)$. Region E contains a ligand-binding pocket buried in a helical globular domain where agonists or antagonists bind. Region $\mathrm{E}$ is also involved in homologous and heterologous dimerization between receptor monomers, and contains the ligand-dependent activation function 2 (AF-2) domain, to which cofactors and repressors bind $(5,9)$. The $\mathrm{F}$ region is only present in certain NRs and there is marked variation in the sequence; however, the structure and function of this region remain to be fully elucidated.

NRs can be divided into three categories based on the different types of ligands they bind with: Class I includes steroid hormone receptors, including androgen receptor, estrogen receptor and glucocorticoid receptor; class II includes non-steroid hormone receptors, including thyroid hormone receptor, retinoic acid receptor and vitamin $\mathrm{D}$ receptor; and class III includes orphan nuclear hormone receptors, including nuclear receptor subfamily 4 group A member 1 
(Nur77), nuclear receptor subfamily 0 group B member 1 (NR0B1) and nuclear receptor subfamily 0 group B member 2 (NR0B2/SHP) (10). NRs have been crucial in the development of targeted drugs, including BMS-564929 and ketoconazole (11). Currently, $13 \%$ of clinically applied drugs target NRs. Therefore, NRs have been established as useful targets for drug development (12).

\section{Identification of Nur77}

The Nur77 gene (also known as NR4A1/NGFI-B/TR3/NAK-1/ GFRP1/HMR/N10/NP10) is located at chromosome 12 , NC_000012.12 $(52,022,832-52,059,507)$ in humans. Nur77 was first identified in 1988 by Hazel et al (13), having previously identified several immediate early genes expressed during the G0/G1 transition in mouse fibroblasts. The transcriptional activity of these genes is activated following stimulation with serum or growth factors. The nucleotide sequence of one of the cDNA clones, Nur77 (initially termed 3CH77), was revealed to encode a member of the ligand-binding transcription factor superfamily, which included steroids and thyroid hormone receptors (13). Subsequently, the Nur77 rat homolog, nerve growth factor-induced clone B (NGFI-B), was successfully cloned from rat adrenal pheochromocytoma cells (PC-12) by Watson and Milbrandt (14). In the same year, testicular receptor 3, a human homologue of Nur77, was identified by Chang et al (15) from a cDNA library of human prostate cancer cells. An increasing number of studies have shown that this transcription factor is present in various species, and it is recognized as a member of NR subfamily 4 group A $(16,17)$.

\section{Structure, expression and localization of Nur77}

Structure of Nur77. The Nur77 protein consists of 598 amino acids and contains A/B, C, D, and E domains (Fig. 1B) (18). Rehman et al (19) identified two protein subtypes in mice that lack the Nur77 N-terminal domain, and the localization of these isoforms was predicted to be predominantly outside the nucleus. Therefore, $\mathrm{N}$-terminal transactivation domain may be required for the transport of Nur77 from the nucleus to the cytoplasm. The DBD region recognizes the specific NGFI-B response element (NBRE; sequence: AAAGGTCA) in target genes and regulates the expression level of these genes $(20,21)$. In addition, Nur77 and retinoid X receptors (RXRs) form heterologous dimers that can combine with the DR5 response element (sequence: AGGTCA-NNNAA-AGGTCA, N: Any single nucleotide) to regulate the transcription of target genes $(22,23)$. The LBD region of Nur77 is distinct from the typical LBD region present in other NRs. The human Nur77 crystal structure (Protein Data Bank-ID: 2QW4; https://www.rcsb.org/structure/2QW4) shows that the Nur77 LBD region is blocked by hydrophobic residues $(24,25)$. Several NRs have been reported to have a hydrophobic-cleft regulated by helices 3, 5 and 12, which is important for the recruitment of co-activators or co-repressors involved in transcriptional regulation (26). However, it is noteworthy that, this cleft is hydrophilic in the Nur77 protein. Additionally, partial denaturation experiments have revealed that helix 12 is relatively flexible in Nur77 (27). Notably, Moore et al (28) identified NR alternate-site modulators that bind with alternative pockets of a protein, rather than the classical LBD, which may result in a different function.

Expression of Nur77. Nur77 has received specific interest in the scientific community due to its role in apoptosis and cancer. The expression level and location of Nur77 are important for protein function. For example, in cells treated with an n-butylidenephthalide derivative (PCH4), the expression of Nur77 was shown to be increased and the protein migrated from the nucleus to the cytoplasm, which may inhibit the growth of malignant glioma cell growth and induce apoptosis (29). Therefore, $\mathrm{PCH} 4$ may be useful as a novel agent for the treatment of malignant glioma. Subsequently, the overexpression of Nur77 in breast cancer cell xenografts was reported to alter the inflammatory response and increase the risk of metastatic disease in mice (30). Another study suggested that the cytoplasmic expression of Nur77 can induce apoptosis in breast cancer cells (31). Notably, one report demonstrated that Nur77 is involved in the migration of transforming growth factor- $\beta$-induced breast cancer cells (32); furthermore, this process is dependent on p38 $\alpha$ (33). Delgado et al (34) reported that the expression of Nur77 in ovaries was high relative to that in other tissues, with only skeletal muscle and tracheal tissue having higher levels of expression than the ovaries. In normal ovarian tissues, Nur77 is predominantly localized in the nucleus, whereas in ovarian cancer cell lines and tissues from patients with ovarian cancer, Nur77 is present in the cytoplasm and the nucleus. In addition, Nur77 was shown to be expressed at high levels in samples from a subset of patients with high-grade serous ovarian cancer, who had a poor progression-free survival rate (34), suggesting that Nur77 mediates the growth of cancer cells. Furthermore, Zhang et al (35) demonstrated that the expression of Nur77 was markedly reduced in the placenta of women with preeclampsia compared with placental levels during a normal pregnancy, and that Nur77 may also be involved in regulating trophoblast cell motility.

Localization of Nur77. The following section considers what determines the positioning of Nur77 in cells. In normal tissues, Nur77 is located in the nucleus due to the NLS in the protein structure; additionally, nuclear Nur77 acts as a carcinogenic survival factor and promotes the growth of cancer cells. By contrast, Nur77 is a potent death promoter when it is located in the mitochondria, where it binds and induces conformational changes in B-cell lymphoma-2 (Bcl-2) protein, triggering the release of cytochrome $c$ and apoptosis (36). There are several potential explanations for this dual function of Nur77. Firstly, the classical hydrophobic nuclear export signal is crucial in Nur77. RXR $\alpha$ acts as an active partner in transporting Nur77 from the nucleus to the cytoplasm, however, translocation of the RXR $\alpha /$ Nur77 heterodimer appears to largely depend on the cell type and stimulus $(37,38)$. Furthermore, studies have reported that the phosphorylation status of Nur77 is vital in the nuclear to cytoplasmic translocation of Nur77, which is regulated by the mitogen-activated protein kinase (MAPK)/c-Jun N-terminal kinase and protein kinase B (Akt) pathways $(34,39,40)$. During oxidative stress-induced cell death in SH-SY5Y cells, Nur77 is upregulated and is translocated from the nucleus to the cytosol and mitochondria (41). Additionally, agents that induce the migration of Nur77 from the nucleus to the mitochondria 


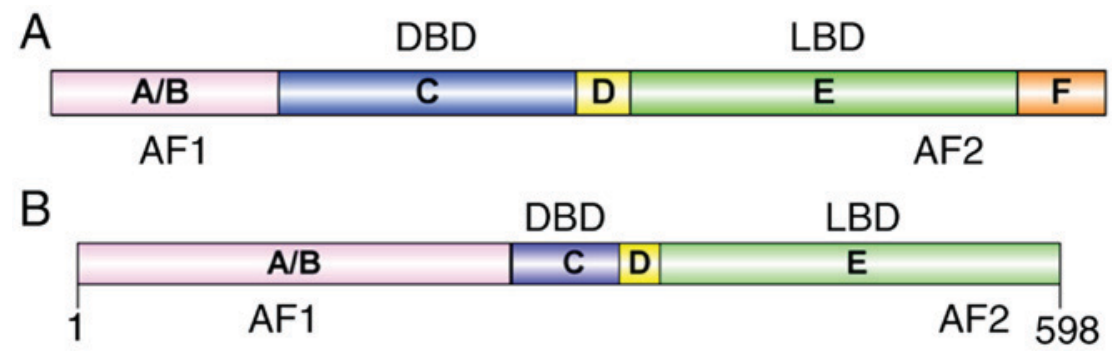

Figure 1. NR structure. (A) Schematic structure of NR. The structure of NRs is divided into five regions: A/B, C, D, E and F. The A/B region termed N-terminal transactivation domain contains $\mathrm{AF}-1$; the $\mathrm{C}$ region is termed the conserved $\mathrm{DBD}$; the $\mathrm{D}$ region is a flexible hinge region; the $\mathrm{E}$ region is termed the $\mathrm{C}$-terminal LBD and contains AF-2; the F region exists in certain NRs and its function remains to be fully elucidated. (B) Schematic structure of Nur77. The LBD region is likely to be between amino acids 351 and 598 (Protein Data Bank: 3V3E). NR, nuclear receptor; DBD, DNA binding domain; LBD, ligand binding domain; AF-1, ligand-independent activation function 1; AF-2, ligand-dependent activation function 2.

effectively induce the apoptosis of cancer cells. For example, following glutamate treatment, Nur77 is translocated from the nucleus to the cytosol and mitochondria in rat cerebellar granule neurons (42). Additionally, by inhibiting the migration of Nur77 from the nucleus to the cytoplasm, 17 $\beta$-estradiol can delay 6-hydroxydopamine-induced apoptosis (43). Endogenous insulin-like growth factor-binding protein 3 has been reported to facilitate the phosphorylation of Nur77 and the export of Nur77 from the nucleus to the cytoplasm, which is vital in intrinsic apoptosis (44). Furthermore, bile acids regulate the expression and intracellular location of Nur77 to control cell survival and death (45).

\section{Genomic and non-genomic functions of the orphan receptor Nur77}

Genomic functions of Nur77. Nur77 is an immediate-early gene and an important transcription factor. The protein was first identified in the monomeric form bound to its response element NBRE. Nur77 has been reported to form heterodimers with RXR/nuclear receptor subfamily 4 group A member 2 (Nurrl)/nuclear receptor subfamily 4 group A member 3 , and these dimers specifically bind to DR5 or NBRE elements (46). Nur77 can also form homodimers and bind to a novel Nur response element, which has a palindromic structure and was identified in the regulatory region of the pro-opiomelanocortin gene. Nur77 is responsive to physiological stimuli in endocrine and lymphoid cells $(21,46)$. Furthermore, the hypothalamic corticotropin-releasing hormone and protein kinase A rapidly increase the nuclear DNA binding activity of Nur77 dimers, but do not affect monomers. The AF-1 domain of Nur77 and the transcriptional mediators/intermediary factor 2, also known as steroid receptor coactivator-2 (SRC-2) glutamine-rich domain are also required for this DNA binding activity (47). Tripartite motif containing 28, also known as KAP-1 or KPIP-1, acts as a transcriptional corepressor for several transcription factors, and it acts synergistically with SRC-2 as an important coactivator of Nur77-dependent transcription (48). There is extensive evidence indicating that Nur77 regulates the expression of several important genes through interaction with specificity protein 1 (Sp1) or specificity protein 4 (Sp4) bound to GC-rich promoters; for example, a Nur77-Sp1-p300 DNA binding complex forms on the GC-rich region close to the survivin promoter to inhibit the expression of survivin, inducing an anticancer effect (49); Nur77 also regulates the expression of $\beta 1$ - and $\beta 3$-integrin via a Nur77/p300/Sp1 complex (50). Integrin gene promoters are GC-rich, and $\alpha 6-, \alpha 5-$, and $\beta 4$-integrins are regulated by Nur77/Sp1/Sp3/Sp4/p300 complexes (51). Binding of the Nur77/Sp4 complex to a GC-rich promoter can enhance the transcription of paired box 3 (PAX3)-forkhead box 01 (FOXO1A); furthermore, the expression of Nur77 regulates $\beta 1$-integrin which, together with PAX3-FOXO1A, contributes to the migration of tumor cells (52). These effects may be exploited for the development of novel antitumor drugs.

There are several downstream genes directly regulated by Nur77, of which a number are involved in the endocrine system, glucose metabolism, the cell cycle, apoptosis, inflammation and other physiological processes. In skeletal muscle, Nur77 directly upregulates the gene expression of glucose transporting protein 4 and promotes the absorption and utilization of glucose by skeletal muscles $(53,54)$. In addition, as a transcription factor, Nur77 typically acts by binding to the NBRE in the promoter regions of target genes. In the liver, Nur77 enhances gluconeogenesis by modulating the expression of genes associated with gluconeogenesis, including glucose-6-phosphatase, fructose bisphosphatase $1 / 2$ and enolase 3 (55-57). Serpin A3 is a member of the serine protease inhibitor family and is mainly synthesized in the liver. It is involved in inflammatory responses and interacts with the Alzheimer's neurotoxic amyloid peptide $\mathrm{A} \beta$ (58). It has been demonstrated that Nur77 regulates SerpinA3 through an NBRE in its promoter region, which may be associated with the role of Nur77 in inflammatory diseases (58). Notably, it has been reported that decanoic acid, a dietary medium-chain fatty acid, can inhibit androgen biosynthesis in NCI-H295R cells, and can reverse endocrine and metabolic aberrations in a rat model of polycystic ovary syndrome; this inhibitory effect may be achieved by the reduced recruitment of Nur77 to the hydroxy- $\delta-5$-steroid dehydrogenase $3 \beta$ - and steroid $\delta$-isomerase 2 (HSD3B2) promoter, which decreases transcription of the HSD3B2 gene and protein expression (59).

Non-genomic functions of Nur77. With the development of science and technology, increasing evidence indicates that Nur77 can also affect the biological functions of other proteins by non-transcriptional functions. In the mitochondria, the Nur77 LBD binds the anti-apoptotic protein Bcl-2, causing a conformation change that exposes the $\mathrm{BH} 3$ domain of $\mathrm{Bcl}-2$; this converts Bcl-2 from an anti-apoptotic protein to a pro-apoptotic protein $(36,60)$. Furthermore, studies have demonstrated that 
p38 $\alpha$ MAPK modulates the Nur77-Bcl-2 apoptotic pathway (61). Another report showed that Nur77 physically interacts with p53, which leads to a decrease in transcriptional activity by inhibiting the acetylation of p53. Nur77 also causes the dissociation of murine double minute 2 (MDM2) from p53, which can prevent p53 from MDM2-induced degradation, increasing the stability of p53 (62). Nur77 can also interact with E1A binding protein p300 and protein arginine methyltransferase 1 (PRMT1). Nur77 suppresses the acetylation of transcription factors induced by p300 (63) and reduces PRMT1 methyltransferase activity. Additionally, PRMT1 increases the DNA binding and transactivation activity of Nur77 in a non-methyltransferase manner (64). Nur77 can bind and sequester liver kinase B1 (LKB1; also known as STK11), which is vital in governing energy homeostasis in the nucleus; sequestering of LKB1 suppresses the phosphorylation of adenosine 5'-monophosphate-activated protein kinase $\alpha(\mathrm{AMPK} \alpha)(65)$. Nur77 also binds to the tuberous sclerosis protein 1 (TSC1)/TSC2 complex to modulate the activity of mammalian target of rapamycin complex 1 (mTORC1), which promotes TSC2 degradation through a proteasome/ubiquitination pathway (66). In addition, the Nur77-mediated activation of mTORC1 is associated with angiotensin II-induced cardiac hypertrophy (66). Inhibiting the interaction between p38 $\alpha$ and Nur77, and inhibiting the p38 $\alpha$-mediated phosphorylation of Nur77 attenuates the lipopolysaccharide-induced hyperinflammatory response (67). The LBD of Nur77 binds to Akt2, and the phosphorylation of Akt 2 can interfere with the migration of Nur77 to the cytoplasm and localization of Nur77 to the mitochondria, which can result in autophagy $(40,68)$.

\section{Compounds that modulate the regulation of Nur77}

In recent years, progress has been made in the treatment of cancer. In addition to the use of chemotherapy to treat cancer, investigations now also focus on developing targeted drugs. Targeted therapy involves the design of a therapeutic drug to interact with a specific target. When the drug enters the body, it interacts specifically with the carcinogenic target to inhibit the growth of tumor cells. Compared with chemotherapy and radiotherapy, targeted therapy can be used to attack tumor cells with reduced effects on healthy cells. This can reduce side effects, thus achieving improved efficacy and improving patient quality of life. There is a demand to identify targets that can be exploited for cancer therapy, and previous studies have shown that Nur77 may be such a target $(69,70)$. However, although there are several reports of drugs that regulate Nur77, there remains limited literature to summarize. In the following section, the drugs reported to be are effective in regulating Nur77 are described.

Ethyl 3,5-dihydroxy-2-(1-oxooctyl)-benzeneacetate (cytosporone B; Csn-B). The octaketide Csn-B (Fig. 2A), which is isolated from the endophytic fungus Dothiorella sp. HTF3 (71), is a natural agonist of nuclear orphan receptor Nur77 with an EC50 of 0.278 nM (72). Csn-B specifically binds to the LBD of Nur77 and enhances Nur77-dependent transactivational activity on target gene transcription (72). Furthermore, Csn-B can induce apoptosis by increasing the expression of Nur77 and promoting the translocation of Nur77 to the cytoplasm to slow xenograft tumor growth (72). Csn-B enhances gluconeogenesis in the mouse liver via increased activation of Nur77. Therefore, Csn-B may be useful in the development of therapeutic agents to treat cancer and hypoglycemia (72). Liu et al (73) synthesized a series of Csn-B analogues. These Csn-B-derived Nur77 agonists are a novel group of potentially potent and effective antitumor agents.

cis-diaminodichloroplatinum (cisplatin). Cisplatin (Fig. 2B) is a chemotherapeutic used to treat various types of cancer. Cisplatin effectively increases the phosphorylation of Nur77 by activating checkpoint kinase 2 and facilitating cross-talk between these two proteins, resulting in apoptosis and the inhibition of intestinal tumorigenesis (74). Additionally, xenografts that originate from the knockdown of Nur77 colon cancer cells are insensitive to cisplatin treatment. Therefore, Nur77 is required for effective cisplatin therapy in colon cancer (74).

Ethyl 2-[2,3,4-trimethoxy-6-(1-octanoyl)-phenyl] acetate (TMPA). TMPA (Fig. 2C) is a Csn-B derivative Nur77 agonist with high affinity. The interaction of TMPA with Nur77 leads to the release and translocation of LKB1 to the cytoplasm, leading to the increased phosphorylation of AMPK $\alpha$ (65). Furthermore, TMPA has been shown to decrease blood glucose and attenuate insulin resistance in type $\mathrm{II} \mathrm{db/db}$ and high-fat diet mice, which was not observed in Nur77 gene-knockout mice (65). These findings indicate that TMPA may have use as a drug for the treatment of metabolic disorders via its effect on Nur77 (65).

N-pentyl 2-[3,5-dihydroxy-2-(1-nonanoyl)-phenyl] acetate (PDNPA). PDNPA (Fig. 2D) targets the LBD of Nur77 and inhibits the interaction between Nur77 and p38 $\alpha$, which can eliminate the p38 $\alpha$-mediated phosphorylation of Nur77 (67). The p38 $\alpha$-mediated phosphorylation of Nur77 impedes the inhibition of nuclear factor $-\kappa \mathrm{B}(\mathrm{NF}-\kappa \mathrm{B})$ by Nur77. As

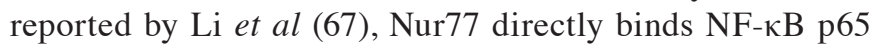
to inhibit its interactions with the $\kappa \mathrm{B}$ response elements in DNA, which inhibits the transcriptional activity of $\mathrm{NF}-\kappa \mathrm{B}$. Additionally, the reduced p38 $\alpha$-Nur77 interaction by PDNPA may alleviate LPS-induced hyperinflammatory responses mediated by Nur77 (67). This finding suggests that modulating p38 $\alpha /$ Nur77-regulated functions may be a novel therapeutic strategy to treat hyperinflammatory responses.

1,3,7-trihydroxy-2,4-diprenylxanthone (CCE9). CCE9 (Fig. 2E), a xanthone compound, is isolated from the Chinese medicinal plant Cratoxylum formosum ssp. pruniflorum (75). CCE9 positively regulates the Nur77/Bcl-2-dependent apoptotic pathway. A previous report suggested that CCE9 can promote the expression of Nur77 and phosphorylation of Bcl-2 in a p38 $\alpha$ MAPK-dependent manner, leading to the cytoplasmic localization and mitochondrial targeting of Nur77 and the Nur77/Bcl-2 interaction (61). The CCE9-induced induction of apoptosis depends on the activation of $\mathrm{p} 38 \alpha$ MAPK, followed by the phosphorylation of Bcl-2 and binding of Bcl-2 with Nur77. Additionally, interference of the expression of p38 $\alpha$ MAPK inhibits the interaction between Bcl-2 and Nur77 (61), and the phosphorylation of Bcl-2 by p38 $\alpha$ MAPK at Ser87 and Thr56 is required for the interaction 
A<smiles>CCCCCCCC(=O)c1c(O)cc(O)cc1CC(=O)OCC</smiles>

Cytosporone B
B<smiles>N[P+](N)(Cl)Cl</smiles>

C<smiles>CCCCCCCC(=O)c1cc(OC)c(OC)c(OC)c1CC(=O)OCC</smiles>

TMPA
D<smiles>CCCCCCCCC(=O)c1c(O)cc(O)cc1CC(=O)OCCCCC</smiles>

PDNPA<smiles>CC(C)=CCc1c(O)c(CC=C(C)C)c2oc3ccc(O)cc3c(=O)c2c1O</smiles>

CCE9
F<smiles>O=C(CBr)c1cc(O)c(O)c(O)c1</smiles>

THPN
G<smiles>CCCC=C1OC(=O)C2=C1CCC=C2</smiles>

Z-Ligustilide<smiles>CC1=C(O)C(=O)C=C2C1=CC=C1[C@@]3(C)CC[C@H](C)[C@]4(C)CC[C@](C)(C(=O)O)C[C@]4(C)[C@H]3CC[C@@]21C</smiles>

Celastrol

Figure 2. Chemical structures of compounds that interact with Nur77. Structures of (A) cytosporone B, (B) cisplatin, (C) TMPA, (D) PDNPA, (E) CCE9, (F) THPN, (G) Z-ligustilide, and (H) celastrol are shown. Cytosporone B, ethyl 3,5-dihydroxy-2-(1-oxooctyl)-benzeneacetate; cisplatin, cis-diaminodichloroplatinum; TMPA, ethyl 2-[2,3,4-trimethoxy-6-(1-octanoyl)-phenyl] acetate; PDNPA, n-pentyl 2-[3,5-dihydroxy-2-(1-nonanoyl)-phenyl] acetate; CCE9, 1,3,7-trihydroxy-2,4-diprenylxanthone; THPN, 1-(3,4,5-trihydroxyphenyl)-nonan-1-one; Z-ligustilide, 3-butylidene-4,5-dihydro-1(3H)-isobenzofuranone; celastrol, 3-hydroxy-9 $\beta, 13 \alpha$-dimethyl-2-oxo-24,25,26-trinoroleana-1(10),3,5,7-tetraen-29-oic acid.

with Nur77 (61). This mechanism of the Nur77-Bcl-2 apoptotic pathway may provide a novel strategy for the development of diverse chemotherapeutic agents.

1-(3,4,5-trihydroxyphenyl)-nonan-1-one (THPN). THPN (Fig. 2F), another Csn-B derivative and Nur77-specific ligand, induces autophagy (76). In melanoma, THPN stimulation was found to increase Nur77 cytoplasmic localization and facilitate its binding with NIP3-like protein X (Nix), a mitochondrial outer membrane protein. The THPN-induced Nix-Nur77 interaction may be the first step in mitochondrial depolarization and autophagic cell death (76). THPN-induced Nur77 transport to the mitochondrial inner membrane may be another critical event in autophagic cell death. Furthermore, THPN inhibits melanoma growth via autophagy, not via the induction of apoptosis (76). This suggests that Nur77 is involved in the regulation of autophagy in melanoma, which may be exploited to develop anticancer therapeutics.
3-Butylidene-4,5-dihydro-1(3H)-isobenzofuranone (Z-ligustilide; Z-LIG). Z-LIG (Fig. 2G) is a phthalide compound and accounts for $>50 \%$ of the volatile oil in Radix Angelica Sinensis (77), which is used to treat gynecological disorders in traditional Chinese medicine and has been reported to have an inhibitory effect on tumors in various types of human cancer, including colon cancer (78) and breast cancer (79). It is well established that autophagy can have a dual role; it can either promote apoptosis or have pro-survival effects, depending on the DNA damage present in cancer cells. For example, autophagy delays apoptotic death in tamoxifen-resistant MCF-7 (MCF-7 ${ }^{\text {TR5 }}$ ) breast cancer cells $(79,80)$. Notably, Z-LIG has been reported to inhibit autophagic flux by inhibiting autophagosome-lysosome fusion; therefore, Z-LIG may be a novel autophagy inhibitor that can induce cell death of tamoxifen-resistant breast cancer cells. Z-LIG has been shown to enhance the efficacy of tamoxifen therapy (79); following Nur77 knockdown, the ability of Z-LIG to sensitize 
tamoxifen-induced cell death was reduced (79). This indicates that Nur77 may be critical in Z-LIG-mediated DNA damage and in the restoration tamoxifen sensitivity in MCF-7 ${ }^{\mathrm{TR} 5}$ cells (79). Together these findings indicate that the effects of Z-LIG and Nur77 may be useful for obtaining novel insights into the mechanisms that mediate tamoxifen resistance in breast cancer.

3-hydroxy-9 $\beta, 13 \alpha$-dimethyl-2-oxo-24,25,26-trinoroleana-1(1 0),3,5,7-tetraen-29-oic acid (celastrol). Celastrol (Fig. $2 \mathrm{H}$ ) is one of the traditional medicinal compounds that may have the potential to be developed as a modern therapeutic agent. It has been reported that celastrol has potent anti-inflammatory activities within several inflammatory diseases and in obesity $(81,82)$; however, the effect of celastrol on inflammatory mechanisms remains to be fully elucidated. It has been reported that celastrol binds Nur77 to inhibit inflammation and induce autophagy in a Nur77-dependent manner. Celastrol induces translocation of Nur77 from the nucleus to the mitochondria, where it is ubiquitinated by tumor necrosis factor receptor-associated factor 2, a scaffold protein, and E3 ubiquitin ligase; these are important for inflammatory signaling (83). The ubiquitinated Nur77 binds to p62/sequestosome 1, which results in sensitivity to autophagy under inflammatory conditions.

As inflammatory mitochondrial dysfunction causes various diseases and types of cancer, these pathways may provide directions for the investigation of cancer and development of novel therapies. Celastrol is a promising drug within this class of therapeutics that acts by targeting Nur77 (83).

Bisindole methane (DIM) compounds. Indole compounds are bioactive components present in cruciferous plants. In relatively low $\mathrm{pH}$ environments, indoles can be converted into various polymers, of which DIM is the most important class. Increasing studies have reported that DIM and its derivatives can specifically bind to and activate multiple nuclear receptors, including Nur77 and Nurr1, to regulate signaling pathways. 1,1-Bis (3-indolyl)-1-(p-substituted phenyl) methane (C-DIM) compounds have significant antitumor activity and low toxicity in cancer cells. Treating athymic nude bearing A549-derived metastases with two C-DIM analogs, DIM-C-pPhOCH3 (C-DIM-5) and DIM-C-pPhOH (C-DIM-8), resulted in significant regression of lung tumors. Furthermore, the effects of the two analogues were different; C-DIM-5 inhibited the cell cycle from the G0/G1 phase to the $\mathrm{S}$ phase, and C-DIM- 8 induced A549 cell apoptosis (84). Additionally, C-DIM-5 and C-DIM-8 have been identified as prototypical activators and antagonists of Nur77, respectively $(49,85)$. C-DIM-5 has been used as a prototype activator for Nur77 in a transactivation assay using the GAL4-Nur77/GAL4-reactive element reporter assay system. However, subsequent investigations with human GAL4-Nur77 showed minimal transactivation of C-DIM-5 (84,86). C-DIM-8 inhibits the activation of Nur77 in pancreatic cancer and lung cancer cells, resulting in the inhibition of cell growth and induction of apoptosis, with results similar to those observed following RNA interference-induced Nur77 (49,87). In addition, C-DIM-5 inhibits the growth and induces apoptosis of UC-5 and KU7 bladder cancer cells (88). The high-affinity interaction between C-DIM-8 and the analogous compounds in the ligand-binding pocket of Nur77 reduces the growth of colon cancer cells, induces cell apoptosis and decreases the expression of survivin and other Sp1-regulated genes (87). Furthermore, treatment of pancreatic, colon and breast cancer cells with C-DIM- 8 or DIM-C-pPhCO2Me resulted in an effect similar to the knockdown of Nur77, with reduced the expression of $\beta 1$-integrin, migration and adhesion. A high expression of $\beta 1$-integrin is a poor prognostic factor for patients with colon and pancreatic cancer, and this protein is important in cell migration and invasion $(50,89,90)$. Additionally, treatment of rhabdomyosarcoma cells, and of ACHN and 786-O renal cell lines with C-DIM-8 and DIM-C-pPhCO2Me was shown to inhibit tumor cell growth and induce apoptosis $(91,92)$. In summary, diindolylmethane analogues bind Nur77 and are Nur77 antagonists in various types of cancer cells, which may provide novel avenues for anticancer treatment.

\section{Conclusions and perspectives}

Nur77 is a relatively well-researched orphan nuclear receptor, and the majority of current studies focus on identifying ligands that regulate the function of Nur77 under pathological conditions. The literature suggests that the orphan nuclear receptor Nur77 is important in cancer, including in colorectal cancer $(93,94)$, androgen-induced bladder cancer $(95)$ and lung cancer (96). In general, although the endogenous ligand for the Nur77 receptor has not been identified, studies have identified structurally diverse compounds that bind to and activate or inactivate Nur77, induce the transfer of Nur77 from the nucleus to the cytoplasm, and can regulate associated diseases. These compounds include Csn-B, cisplatin, celastrol, Z-LIG and DIM compounds. Cisplatin is already an antitumor chemotherapeutic drug. Cytosporone B is currently being assessed for anticancer activates; however, its detailed biological effects and potential side effects require investigation. In addition, whether there are other factors that influence the expression and location of Nur77, the mechanism of action of Nur77 as a potential regulator of inflammation, and the specific association between Nur77 and apoptosis and autophagy remain to be fully elucidated. Furthermore, the continued investigation of compounds that can modulate Nur77 is required, followed by the development and performing of clinical trials using these compounds to evaluate their potential for therapeutic use. In conclusion, the orphan nuclear receptor Nur77 is a promising research target for investigating cancer mechanisms and prospective therapies. It is important to understand the structure, function, expression and localization of Nur77, and to screen and develop compounds that act as agonists or antagonists and may have clinical applications.

\section{Acknowledgements}

Not applicable.

\section{Funding}

This study was supported by grants from the National Natural Science Foundation (grant no. 31500616) and the Natural Science Foundation of Fujian Province (grant no. 2017J01445). 


\section{Availability of data and materials}

Not applicable.

\section{Authors' contributions}

LW and LC designed and supervised the project and wrote the manuscript.

\section{Ethics approval and consent to participate}

Not applicable.

\section{Patient consent for publication}

Not applicable.

\section{Competing interests}

The authors declare that they have no competing interests.

\section{References}

1. Mangelsdorf DJ, Thummel C, Beato M, Herrlich P, Schütz G, Umesono K, Blumberg B, Kastner P, Mark M, Chambon P and Evans RM: The nuclear receptor superfamily: The second decade. Cell 83: 835-839, 1995.

2. Margolis RN and Christakos S: The nuclear receptor superfamily of steroid hormones and vitamin D gene regulation. An update. Ann N Y Acad Sci 1192: 208-214, 2010.

3. Robinson-Rechavi M, Escriva Garcia $\mathrm{H}$ and Laudet V: The nuclear receptor superfamily. J Cell Sci 116: 585-586, 2003.

4. Giguere V: Structure and function of the nuclear receptor superfamily for steroid, thyroid hormone and retinoic acid. Genet Eng (N Y) 12: 183-200, 1990.

5. Brelivet Y, Rochel N and Moras D: Structural analysis of nuclear receptors: From isolated domains to integral proteins. Mol Cell Endocrinol 348: 466-473, 2012.

6. McEwan IJ: The Nuclear receptor superfamily at thirty. Methods Mol Biol 1443: 3-9, 2016.

7. Germain P, Staels B, Dacquet C, Spedding M and Laudet V: Overview of nomenclature of nuclear receptors. Pharmacol Rev 58: 685-704, 2006

8. Rastinejad F, Huang P, Chandra V and Khorasanizadeh S: Understanding nuclear receptor form and function using structural biology. J Mol Endocrinol 51: T1-T21, 2013.

9. Chandra V, Huang P, Hamuro Y, Raghuram S, Wang Y, Burris TP and Rastinejad F: Structure of the intact PPAR-gammaRXR-nuclear receptor complex on DNA. Nature 456: 350-356, 2008 .

10. Olefsky JM: Nuclear receptor minireview series. J Biol Chem 276: 36863-36864, 2001

11. Chen T: Nuclear receptor drug discovery. Curr Opin Chem Biol 12: 418-426, 2008

12. Chen T: Overcoming drug resistance by regulating nuclear receptors. Adv Drug Deliv Rev 62: 1257-1264, 2010.

13. Hazel TG, Nathans D and Lau LF: A gene inducible by serum growth factors encodes a member of the steroid and thyroid hormone receptor superfamily. Proc Natl Acad Sci USA 85 : 8444-8448, 1988

14. Watson MA and Milbrandt J: The NGFI-B gene, a transcriptionally inducible member of the steroid receptor gene superfamily: Genomic structure and expression in rat brain after seizure induction. Mol Cell Biol 9: 4213-4219, 1989.

15. Chang C, Kokontis J, Liao SS and Chang Y: Isolation and characterization of human TR3 receptor: A member of steroid receptor superfamily. J Steroid Biochem 34: 391-395, 1989.

16. Hwang DS, Lee BY, Kim HS, Lee MC, Kyung DH, Om AS Rhee JS and Lee JS: Genome-wide identification of nuclear receptor (NR) superfamily genes in the copepod Tigriopus japonicus. BMC Genomics 15: 993, 2014.
17. Sharma Y, Chilamakuri CS, Bakke $M$ and Lenhard B: Computational characterization of modes of transcriptional regulation of nuclear receptor genes. PLoS One 9: e88880, 2014.

18. Kurakula K, Koenis DS, van Tiel CM and de Vries CJ: NR4A nuclear receptors are orphans but not lonesome. Biochim Biophys Acta 1843: 2543-2555, 2014.

19. Rehman SU, Sarwar T, Husain MA, Ishqi HM and Tabish M: Identification of two novel isoforms of mouse NUR77 lacking N-terminal domains. IUBMB Life 69: 106-114, 2017.

20. Wilson TE, Fahrner TJ and Milbrandt J: The orphan receptors NGFI-B and steroidogenic factor 1 establish monomer binding as a third paradigm of nuclear receptor-DNA interaction. Mol Cell Biol 13: 5794-5804, 1993.

21. Maira M, Martens C, Philips A and Drouin J: Heterodimerization between members of the Nur subfamily of orphan nuclear receptors as a novel mechanism for gene activation. Mol Cell Biol 19: 7549-7557, 1999.

22. Forman BM, Goode E, Chen J, Oro AE, Bradley DJ, Perlmann T, Noonan DJ, Burka LT, McMorris T, Lamph WW, et al: Identification of a nuclear receptor that is activated by farnesol metabolites. Cell 81: 687-693, 1995.

23. Perlmann T and Jansson L: A novel pathway for vitamin A signaling mediated by RXR heterodimerization with NGFI-B and NURR1. Genes Dev 9: 769-782, 1995.

24. Flaig R, Greschik H, Peluso-Iltis C and Moras D: Structural basis for the cell-specific activities of the NGFI-B and the Nurrl ligand-binding domain. J Biol Chem 280: 19250-19258, 2005.

25. Michiels P, Atkins K, Ludwig C, Whittaker S, van Dongen M and Günther U: Assignment of the orphan nuclear receptor Nurrl by NMR. Biomol NMR Assign 4: 101-105, 2010.

26. Wansa KD, Harris JM and Muscat GE: The activation function-1 domain of Nur77/NR4A1 mediates trans-activation, cell specificity, and coactivator recruitment. J Biol Chem 277: 33001-33011, 2002.

27. Lanig H, Reisen F, Whitley D, Schneider G, Banting L and Clark T: In silico adoption of an orphan nuclear receptor NR4A1. PLoS One 10: e0135246, 2015.

28. Moore TW, Mayne CG and Katzenellenbogen JA: Minireview: Not picking pockets: Nuclear receptor alternate-site modulators (NRAMs). Mol Endocrinol 24: 683-695, 2010.

29. Chang LF, Lin PC, Ho LI, Liu PY, Wu WC, Chiang IP, Chang HW, Lin SZ, Harn YC, Harn HJ and Chiou TW: Overexpression of the orphan receptor Nur77 and its translocation induced by $\mathrm{PCH} 4$ may inhibit malignant glioma cell growth and induce cell apoptosis. J Surg Oncol 103: 442-450, 2011.

30. Holmes WF, Soprano DR and Soprano KJ: Early events in the induction of apoptosis in ovarian carcinoma cells by CD437: Activation of the p38 MAP kinase signal pathway. Oncogene 22: 6377-6386, 2003.

31. Niu G, Lu L, Gan J, Zhang D, Liu J and Huang G: Dual roles of orphan nuclear receptor TR3/Nur77/NGFI-B in mediating cell survival and apoptosis. Int Rev Cell Mol Biol 313: 219-258, 2014.

32. Zhou F, Drabsch Y, Dekker TJ, de Vinuesa AG, Li Y, Hawinkels LJ, Sheppard KA, Goumans MJ, Luwor RB, de Vries CJ, et al: Nuclear receptor NR4A1 promotes breast cancer invasion and metastasis by activating TGF- $\beta$ signalling. Nat Commun 5: 3388, 2014.

33. Hedrick E and Safe S: Transforming growth factor

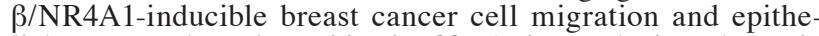
lial-to-mesenchymal transition is $\mathrm{p} 38 \alpha$ (Mitogen-Activated Protein Kinase 14) dependent. Mol Cell Biol 37: pii: e00306-17, 2017.

34. Delgado E, Boisen MM, Laskey R, Chen R, Song C, Sallit J, Yochum ZA, Andersen CL, Sikora MJ, Wagner J, et al: High expression of orphan nuclear receptor NR4A1 in a subset of ovarian tumors with worse outcome. Gynecol Oncol 141: 348-356, 2016.

35. Zhang X, Yan G, Diao Z, Sun H and Hu Y: NUR77 inhibits the expression of TIMP2 and increases the migration and invasion of HTR-8/SVneo cells induced by CYR61. Placenta 33: 561-567, 2012.

36. Lin B, Kolluri SK, Lin F, Liu W, Han YH, Cao X, Dawson MI, Reed JC and Zhang XK: Conversion of Bcl-2 from protector to killer by interaction with nuclear orphan receptor Nur77/TR3. Cell 116: 527-540, 2004.

37. Cao X, Liu W, Lin F, Li H, Kolluri SK, Lin B, Han YH, Dawson MI andZhang XK: Retinoid Xreceptor regulates Nur77/TR3-dependent apoptosis [corrected] by modulating its nuclear export and mitochondrial targeting. Mol Cell Biol 24: 9705-9725, 2004.

38. Zhang XK: Targeting Nur77 translocation. Expert Opin Ther Targets 11: 69-79, 2007.

39. Han YH, Cao X, Lin B, Lin F, Kolluri SK, Stebbins J, Reed JC, Dawson MI and Zhang XK: Regulation of Nur77 nuclear export by c-Jun N-terminal kinase and Akt. Oncogene 25: 2974-2986, 2006. 
40. Chen HZ, Zhao BX, Zhao WX, Li L, Zhang B and Wu Q: Akt phosphorylates the TR3 orphan receptor and blocks its targeting to the mitochondria. Carcinogenesis 29: 2078-2088, 2008.

41. No H, Bang Y, Lim J, Kim SS, Choi HS and Choi HJ: Involvement of induction and mitochondrial targeting of orphan nuclear receptor Nur77 in 6-OHDA-induced SH-SY5Y cell death. Neurochem Int 56: 620-626, 2010.

42. Boldingh Debernard KA, Mathisen GH and Paulsen RE: Differences in NGFI-B, Nurr1, and NOR-1 expression and nucleocytoplasmic translocation in glutamate-treated neurons. Neurochem Int 61: 79-88, 2012.

43. Renaud J, Chiasson K, Bournival J, Rouillard C and Martinoli MG: $17 \beta$-estradiol delays 6-OHDA-induced apoptosis by acting on Nur77 translocation from the nucleus to the cytoplasm. Neurotox Res 25: 124-134, 2014

44. Agostini-Dreyer A, Jetzt AE, Stires H and Cohick WS: Endogenous IGFBP-3 mediates intrinsic apoptosis through modulation of Nur77 phosphorylation and nuclear export. Endocrinology 156: 4141-4151, 2015.

45. Hu Y, Chau T, Liu HX, Liao D, Keane R, Nie Y, Yang H and Wan YJ: Bile acids regulate nuclear receptor (Nur77) expression and intracellular location to control proliferation and apoptosis Mol Cancer Res 13: 281-292, 2015.

46. Drouin J, Maira M and Philips A: Novel mechanism of action for Nur77 and antagonism by glucocorticoids: A convergent mechanism for $\mathrm{CRH}$ activation and glucocorticoid repression of POMC gene transcription. J Steroid Biochem Mol Biol 65: 59-63, 1998.

47. Maira M, Martens C, Batsché E, Gauthier Y and Drouin J: Dimer-specific potentiation of NGFI-B (Nur77) transcriptional activity by the protein kinase A pathway and AF-1-dependent coactivator recruitment. Mol Cell Biol 23: 763-776, 2003.

48. Rambaud J, Desroches J, Balsalobre A and Drouin J: TIFlbeta/KAP-1 is a coactivator of the orphan nuclear receptor NGFI-B/Nur77. J Biol Chem 284: 14147-14156, 2009.

49. Lee SO, Abdelrahim M, Yoon K, Chintharlapalli S, Papineni S, Kim K, Wang H and Safe S: Inactivation of the orphan nuclear receptor TR3/Nur77 inhibits pancreatic cancer cell and tumor growth. Cancer Res 70: 6824-6836, 2010.

50. Hedrick E, Lee SO, Doddapaneni R, Singh M and Safe S: NR4A1 antagonists inhibit $\beta 1$-integrin-dependent breast cancer cell migration. Mol Cell Biol 36: 1383-1394, 2016.

51. Hedrick E, Li X and Safe S: Penfluridol represses integrin expression in breast cancer through induction of reactive oxygen species and downregulation of Sp transcription factors. Mol Cancer Ther 16: 205-216, 2017.

52. Lacey A, Rodrigues-Hoffman A and Safe S: PAX3-FOXO1A expression in rhabdomyosarcoma is driven by the targetable nuclear receptor NR4A1. Cancer Res 77: 732-741, 2017.

53. Chao LC, Zhang Z, Pei L, Saito T, Tontonoz P and Pilch PF: Nur77 coordinately regulates expression of genes linked to glucose metabolism in skeletal muscle. Mol Endocrinol 21: 2152-2163, 2007.

54. Kanzleiter T, Preston E, Wilks D, Ho B, Benrick A, Reznick J, Heilbronn LK, Turner N and Cooney GJ: Overexpression of the orphan receptor Nur77 alters glucose metabolism in rat muscle cells and rat muscle in vivo. Diabetologia 53: 1174-1183, 2010.

55. Pei L, Waki H, Vaitheesvaran B, Wilpitz DC, Kurland IJ and Tontonoz P: NR4A orphan nuclear receptors are transcriptional regulators of hepatic glucose metabolism. Nat Med 12: 1048-1055, 2006.

56. Pols TW, Ottenhoff R, Vos M, Levels JH, Quax PH, Meijers JC, Pannekoek H, Groen AK and de Vries CJ: Nur77 modulates hepatic lipid metabolism through suppression of SREBP1c activity. Biochem Biophys Res Commun 366: 910-916, 2008

57. Zhao Y and Bruemmer D: NR4A orphan nuclear receptors: Transcriptional regulators of gene expression in metabolism and vascular biology. Arterioscler Thromb Vasc Biol 30: 1535-1541, 2010.

58. Zhao Y, Liu Y and Zheng D: Alpha 1-antichymotrypsin/SerpinA3 is a novel target of orphan nuclear receptor Nur77. FEBS J 275: $1025-1038,2008$.

59. Lee BH, Indran IR, Tan HM, Li Y, Zhang Z, Li J and Yong EL: A dietary medium-chain fatty acid, decanoic acid, inhibits recruitment of Nur77 to the HSD3B2 promoter in vitro and reverses endocrine and metabolic abnormalities in a rat model of polycystic ovary syndrome. Endocrinology 157: 382-394, 2016

60. Kolluri SK, Zhu X,Zhou X, Lin B, Chen Y, Sun K, Tian X, Town J, Cao X, Lin F, et al: A short Nur77-derived peptide converts Bcl-2 from a protector to a killer. Cancer Cell 14: 285-298, 2008.
61. Liu J, Wang GH, Duan YH, Dai Y, Bao Y, Hu M, Zhou YQ, Li M, Jiang F, Zhou H, et al: Modulation of the Nur77-Bcl-2 apoptotic pathway by p38alpha MAPK. Oncotarget 8: 69731-69745, 2017.

62. Zhao BX, Chen HZ, Lei NZ, Li GD, Zhao WX, Zhan YY, Liu B Lin SC and Wu Q: p53 mediates the negative regulation of MDM2 by orphan receptor TR3. EMBO J 25: 5703-5715, 2006

63. Li GD, Fang JX, Chen HZ, Luo J, Zheng ZH, Shen YM and Wu Q: Negative regulation of transcription coactivator p300 by orphan receptor TR3. Nucleic Acids Res 35: 7348-7359, 2007.

64. Lei NZ, Zhang XY, Chen HZ, Wang Y, Zhan YY, Zheng ZH, Shen YM and Wu Q: A feedback regulatory loop between methyltransferase PRMT1 and orphan receptor TR3. Nucleic Acids Res 37: 832-848, 2009.

65. Zhan YY, Chen Y, Zhang Q, Zhuang JJ, Tian M, Chen HZ, Zhang LR, Zhang HK, He JP, Wang WJ, et al: The orphan nuclear receptor Nur77 regulates LKB1 localization and activates AMPK. Nat Chem Biol 8: 897-904, 2012.

66. Wang RH, He JP, Su ML, Luo J, Xu M, Du XD, Chen HZ, Wang WJ, Wang Y, Zhang N, et al: The orphan receptor TR3 participates in angiotensin II-induced cardiac hypertrophy by controlling mTOR signalling. EMBO Mol Med 5: 137-148, 2013.

67. Li L, Liu Y, Chen HZ, Li FW, Wu JF, Zhang HK, He JP, Xing YZ, Chen Y, Wang WJ, et al: Impeding the interaction between Nur77 and p38 reduces LPS-induced inflammation. Nat Chem Biol 11: 339-346, 2015.

68. Wang WJ, Wang Y, Hou PP, Li FW, Zhou B, Chen HZ, Bian XL, Cai QX, Xing YZ, He JP, et al: Induction of autophagic death in cancer cells by agonizing TR3 and attenuating Akt2 activity. Chem Biol 22: 1040-1051, 2015.

69. To SK, Zeng JZ and Wong AS: Nur77: A potential therapeutic target in cancer. Expert Opin Ther Targets 16: 573-585, 2012.

70. Zeng Y, Ye X, Liao D, Huang S, Mao H, Zhao D and Zeng H: Orphan nuclear receptor TR3/Nur77 is a specific therapeutic target for hepatic cancers. J Clin Exp Oncol 6: pii: 184, 2017.

71. Brady SF, Wagenaar MM, Singh MP, Janso JE and Clardy J: The cytosporones, new octaketide antibiotics isolated from an endophytic fungus. Org Lett 2: 4043-4046, 2000

72. Zhan Y, Du X, Chen H, Liu J, Zhao B, Huang D, Li G, Xu Q, Zhang M, Weimer BC, et al: Cytosporone B is an agonist for nuclear orphan receptor Nur77. Nat Chem Biol 4: 548-556, 2008.

73. Liu JJ, Zeng HN, Zhang LR, Zhan YY, Chen Y, Wang Y, Wang J, Xiang SH, Liu WJ, Wang WJ, et al: A unique pharmacophore for activation of the nuclear orphan receptor Nur77 in vivo and in vitro. Cancer Res 70: 3628-3637, 2010.

74. Yao LM, He JP, Chen HZ, Wang Y, Wang WJ, Wu R, Yu CD and Wu Q: Orphan receptor TR3 participates in cisplatin-induced apoptosis via Chk2 phosphorylation to repress intestinal tumorigenesis. Carcinogenesis 33: 301-311, 2012.

75. Duan YH, Dai Y, Wang GH, Zhang X, Chen HF, Chen JB, Yao XS and Zhang XK: Bioactive xanthones from the stems of Cratoxylum formosum ssp. pruniflorum. J Nat Prod 73: 1283-1287, 2010

76. Wang WJ, Wang Y, Chen HZ, Xing YZ, Li FW, Zhang Q, Zhou B, Zhang HK, Zhang J, Bian XL, et al: Orphan nuclear receptor TR3 acts in autophagic cell death via mitochondrial signaling pathway. Nat Chem Biol 10: 133-140, 2014.

77. Zeng Q, Jia YW, Xu PL, Xiao MW, Liu YM, Peng SL and Liao X: Quick and selective extraction of Z-ligustilide from Angelica sinensis using magnetic multiwalled carbon nanotubes. J Sep Sci 38: 4269-4275, 2015.

78. Kan WL, Cho CH, Rudd JA andLin G: Study of the anti-proliferative effects and synergy of phthalides from Angelica sinensis on colon cancer cells. J Ethnopharmacol 120: 36-43, 2008

79. Qi H, Jiang Z, Wang C, Yang Y, Li L, He H and Yu Z: Sensitization of tamoxifen-resistant breast cancer cells by Z-ligustilide through inhibiting autophagy and accumulating DNA damages. Oncotarget 8: 29300-29317, 2017

80. Abedin MJ, Wang D, McDonnell MA, Lehmann U and Kelekar A: Autophagy delays apoptotic death in breast cancer cells following DNA damage. Cell Death Differ 14: 500-510, 2007.

81. Greenhill C: Celastrol identified as a leptin sensitizer and potential novel treatment for obesity. Nat Rev Endocrinol 11: 444, 2015

82. Liu J, Lee J, Salazar Hernandez MA, Mazitschek R and Ozcan U: Treatment of obesity with celastrol. Cell 161: 999-1011,2015.

83. Hu M, Luo Q, Alitongbieke G, Chong S, Xu C, Xie L, Chen X, Zhang D, Zhou Y, Wang Z, et al: Celastrol-induced Nur77 interaction with TRAF2 alleviates inflammation by promoting mitochondrial ubiquitination and autophagy. Mol Cell 66: 141-153.e6, 2017. 
84. Andey T, Patel A, Jackson T, Safe S and Singh M: 1,1-Bis (3'-indolyl)-1-(p-substitutedphenyl)methane compounds inhibit lung cancer cell and tumor growth in a metastasis model. Eur J Pharm Sci 500: 227-241, 2013.

85. Yoon K, Lee SO, Cho SD, Kim K, Khan S and Safe S: Activation of nuclear TR3 (NR4A1) by a diindolylmethane analog induces apoptosis and proapoptotic genes in pancreatic cancer cells and tumors. Carcinogenesis 32: 836-842, 2011.

86. Cho SD, Yoon K, Chintharlapalli S, Abdelrahim M, Lei P Hamilton S, Khan S, Ramaiah SK and Safe S: Nur77 agonists induce proapoptotic genes and responses in colon cancer cells through nuclear receptor-dependent and nuclear receptor-independent pathways. Cancer Res 67: 674-683, 2007.

87. Lee SO, Li X, Hedrick E, Jin UH, Tjalkens RB, Backos DS, Li L, Zhang Y, Wu Q and Safe S: Diindolylmethane analogs bind NR4A1 and are NR4A1 antagonists in colon cancer cells. Mol Endocrinol 28: 1729-1739, 2014.

88. Cho SD, Lee SO, Chintharlapalli S, Abdelrahim M, Khan S, Yoon K, Kamat AM and Safe S: Activation of nerve growth factor-induced B alpha by methylene-substituted diindolylmethanes in bladder cancer cells induces apoptosis and inhibits tumor growth. Mol Pharmacol 77: 396-404, 2010.

89. Hedrick E, Lee SO and Safe S: The nuclear orphan receptor NR4A1 regulates $\beta 1$-integrin expression in pancreatic and colon cancer cells and can be targeted by NR4A1 antagonists. Mol Carcinog 56: 2066-2075, 2017.
90. Hedrick E, Lee SO, Doddapaneni R, Singh M and Safe S: Nuclear receptor $4 \mathrm{~A} 1$ as a drug target for breast cancer chemotherapy. Endocr Relat Cancer 22: 831-840, 2015.

91. Lacey A, Hedrick E, Li X, Patel K, Doddapaneni R, Singh M and Safe S: Nuclear receptor 4A1 (NR4A1) as a drug target for treating rhabdomyosarcoma (RMS). Oncotarget 7: 31257-31269, 2016.

92. Hedrick E, Lee SO, Kim G, Abdelrahim M, Jin UH, Safe S and Abudayyeh A: Nuclear receptor 4A1 (NR4A1) as a drug target for renal cell adenocarcinoma. PLoS One 10: e0128308, 2015.

93. Wang JR, Gan WJ, Li XM, Zhao YY, Li Y, Lu XX, Li JM and Wu H: Orphan nuclear receptor Nur77 promotes colorectal cancer invasion and metastasis by regulating MMP-9 and E-cadherin. Carcinogenesis 35: 2474-2484, 2014.

94. To SK, Zeng WJ, Zeng JZ and Wong AS: Hypoxia triggers a Nur77- $\beta$-catenin feed-forward loop to promote the invasive growth of colon cancer cells. Br J Cancer 110: 935-945, 2014.

95. Wu J, Liu J, Jia R and Song H: Nur77 inhibits androgen-induced bladder cancer growth. Cancer Invest 31: 654-660, 2013.

96. Wohlkoenig C, Leithner K, Olschewski A, Olschewski H and Hrzenjak A: TR3 is involved in hypoxia-induced apoptosis resistance in lung cancer cells downstream of HIF-1 $\alpha$. Lung Cancer 111: 15-22, 2017.

This work is licensed under a Creative Commons Attribution-NonCommercial-NoDerivatives 4.0 International (CC BY-NC-ND 4.0) License. 